\title{
Incoherent pair background processes with full polarizations at the ILC
}

\author{
A. $\operatorname{Hartin}^{1 *}$ \\ Oxford University - Physics Department \\ Keble Road, Oxford OX3 7TF- UK
}

\begin{abstract}
Incoherent background pair production processes are studied with respect to full polarizations of all states. Real initial photon polarizations are obtained via a QED calculation of the beamstrahlung process. Virtual photon polarizations are related to the electric field of the colliding bunches at the point of pair production. An explicit expression for the virtual photon polarization vector is developed and found to have no circular polarization component. Pair polarization states are highly dependent on initial state circular polarization and are consequently produced almost unpolarized. The Breit-Wheeler cross-section with full polarizations is calculated and coded into the CAIN pair generator program. Numerical evaluations of the ILC, operating with the seven proposed collider parameter sets, shows that there are $10-20 \%$ less low energy pairs than previously thought. Collider luminosity, as calculated by CAIN, remains the same.
\end{abstract}

\section{INTRODUCTION}

The International Linear Collider will collide polarized particle bunches to produce physics processes of interest and background events. The polarization states of both the bunch particles and the collective field of the bunch are important parameters affecting cross-sections and hence final particle states. It is important to calculate precisely the full polarization effects and this will be done here for incoherent background pair processes resulting from beam-beam interactions. A theoretical exposition will be required and the program CAIN will be modified for full polarizations in order to produce numerical results.

There are three incoherent background pair processes considered to produce background $e^{ \pm}$pairs at the ILC. The Breit-Wheeler process, has two real photons in its initial state. The Bethe-Heitler and Landau-Lifshitz processes contain initial states of one and two virtual photons respectively. The flux of virtual photons is established using the well known Weizsacker-Williams virtual photon approximation. In all three processes, once the initial particle states are established, the same BreitWheeler cross-section is used to calculate the total number of background pair particles produced via beam-beam collisions. Three important theoretical expressions are necessary; the polarization of initial real and virtual photon states, the Breit-Wheeler cross-section containing all polarization states, and the polarization of final states.

\section{THEORY}

Real photons engaged in pair production processes are produced via the beamstrahlung process. The present version of CAIN contains full polarizations for this process and the representation in Stokes parameters is given in equation 5.510 of the CAIN manual [1]. An expression for virtual photon polarization states in terms of Stokes parameters has to be developed. The Stokes parameter of a

\footnotetext{
*This work is supported in part by the Commission of the European Communities under the 6th Framework Programme "Structuring the European Research Area", contract number RIDS-011899.
}

LCWS/ILC 2007 


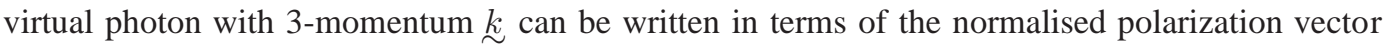
$\left(\hat{e}_{x}, \hat{e}_{y}, \hat{k}_{z}\right)$ as

$$
\begin{aligned}
& \xi_{1}=\hat{e}_{x} \hat{e}_{y}^{*}+\hat{e}_{y} \hat{e}_{x}^{*} \\
& \xi_{2}=\Im\left[\hat{e}_{y} \hat{e}_{x}^{*}-\hat{e}_{x} \hat{e}_{y}^{*}\right] \\
& \xi_{3}=\hat{e}_{x} \hat{e}_{x}^{*}-\hat{e}_{y} \hat{e}_{y}^{*}
\end{aligned}
$$

To obtain an explicit form for the Stokes parameters it is recognised that the virtual photon polarization vector can be identified with the spectral component $E_{\omega}$ of the electric field of the particle bunch. The expression for $E_{\omega}$ is written down first from the expansion of the electric field $E_{q}$ of a single relativistic particle of speed $v$ in plane waves with wave vector $q=\left(q_{x}, q_{y}, q_{z}\right)$. The electric field of a bunch of $N$ such charges is a product of the Gaussian form factor of the bunch and $E_{q}$ integrated over all wave vector components [2]. The spectral component of the collective field at position $(x, y)$ is written finally

$$
\begin{aligned}
& E_{\omega}^{x, y}=-\frac{i e}{\pi v} \iint \frac{q_{x, y}}{q_{x}^{2}+q_{y}^{2}} F(q) \exp \left(i x q_{x}+i y q_{y}\right) d q_{x} d q_{y} \\
& \text { where } \quad F(q)=N \exp \left[-\frac{1}{2}\left(q_{x} \sigma_{x}\right)^{2}-\frac{1}{2}\left(q_{y} \sigma_{y}\right)^{2}\right]
\end{aligned}
$$

Equation 2 is a difficult Fourier transform over two variables. Progress is made by separating variables via a Taylor expansion of $\frac{1}{q_{x}^{2}+q_{y}^{2}}$. Fourier transforms in $q_{x}$ and $q_{y}$ are then easily calculated and terms in the Taylor expansion turn out to be dependent on a ratio of transverse beam sizes $\frac{\sigma_{y}}{\sigma_{x}}$. For the flat beam envisaged for the ILC, this ratio is very small and only the first term in the Taylor expansion is required. The spectral component of the beam field turns out to be

$$
\begin{aligned}
& E_{\omega}^{x}=-\frac{e}{\pi v} \exp \left(-\frac{x^{2}}{2 \sigma_{x}^{2}}\right) \frac{x}{\sigma_{x}^{3}}\left[\exp \left(-\frac{y^{2}}{2 \sigma_{y}^{2}}\right)+y \sqrt{\frac{\pi}{2}} \operatorname{Erf}\left(\frac{y}{\sqrt{2} \sigma_{y}}\right)\right] \\
& E_{\omega}^{y}=\frac{e}{\pi v} \sqrt{\frac{\pi}{2}} \exp \left(-\frac{x^{2}}{2 \sigma_{x}^{2}}\right) \frac{1}{\sigma_{x}} \operatorname{Erf}\left(\frac{y}{\sqrt{2} \sigma_{y}}\right)
\end{aligned}
$$

The expressions in equation 3 are numerically easy to calculate and are inserted into CAIN. The magnitude of the y component of the bunch field $E_{\omega}^{y}$ is much greater than that of the x component. This is consistent intuitively with the bunch being squeezed in y. Both components are real, rendering $\xi_{2}=0$, meaning that there is no circular polarisation of virtual photons. This is consistent with the constant crossed electromagnetic fields associated with relativistic charges.

In order to take advantage of the full polarisation of initial photons, the full Breit-Wheeler crosssection is required. At present in CAIN the cross-section $\sigma^{\text {circ }}$ is written down only for the product of circular polarisations $\xi_{2} \xi_{2}^{\prime}$ of initial photons $k$ and $k^{\prime}$. The full cross-section $\sigma^{\text {full }}$ is a sum over all polarisation states and functions of final electron energy $\epsilon$ and momentum $p$ [3]. With some algebraic manipulation the two cross-sections can be written in similar form 


$$
\begin{aligned}
& \sigma^{\text {circ }} \propto 2\left(1-h_{2}+\frac{2 \epsilon^{2}-1}{2 \epsilon^{2}}\right) \sinh ^{-1} p+\frac{p}{\epsilon}\left(3 h_{2}-1-\frac{1}{\epsilon^{2}}\right) \\
& \begin{aligned}
\sigma^{\text {full }} \propto 2\left(1-h_{2}+\frac{2}{\epsilon^{2}}\left(h_{1}+h_{3}\right)-\frac{h_{3}}{\epsilon^{4}}\right) \sinh ^{-1} p \\
\quad+\frac{p}{\epsilon}\left(3 h_{2}-1-h_{1}-\xi_{3} \xi_{3}^{\prime}-\frac{h_{3}}{\epsilon^{2}}\right)
\end{aligned} \\
& \begin{aligned}
\text { where } \quad h_{1} & =\xi_{1} \xi_{1}^{\prime} \quad h_{2}=\xi_{2} \xi_{2}^{\prime} \\
h_{3} & =1+\xi_{3}+\xi_{3}^{\prime}+\xi_{3} \xi_{3}^{\prime}
\end{aligned}
\end{aligned}
$$

The final analytic expression required for a full investigation of polarization effects, are the polarizations of final states. These are specified by the $e^{ \pm}$polarization vector $\left(\zeta_{1}, \zeta_{2}, \zeta_{3}\right)$ and can be written in terms of a sum over products of initial polarization states and a function $F_{j j^{\prime}}^{i i^{\prime}}$ of 4-vector scalar products [3]

$$
\zeta_{i}=\frac{1}{F} \sum_{i j j^{\prime}} F_{j j^{\prime}}^{i 0} \xi_{j} \xi_{j^{\prime}}^{\prime} \quad \text { where } \quad F=\sum_{j j^{\prime}} F_{j j^{\prime}}^{00} \xi_{j} \xi_{j^{\prime}}^{\prime}
$$

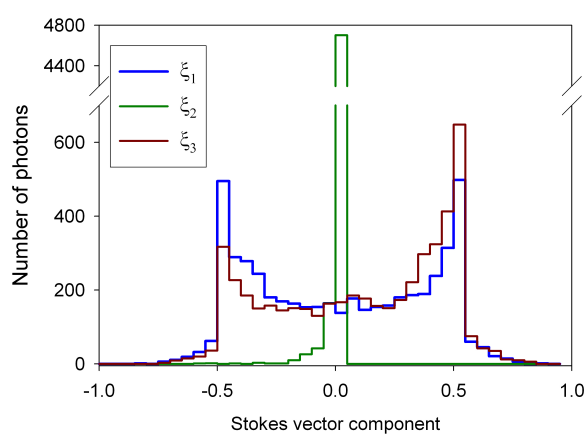

Figure 1: Stokes parameters of inital photons.

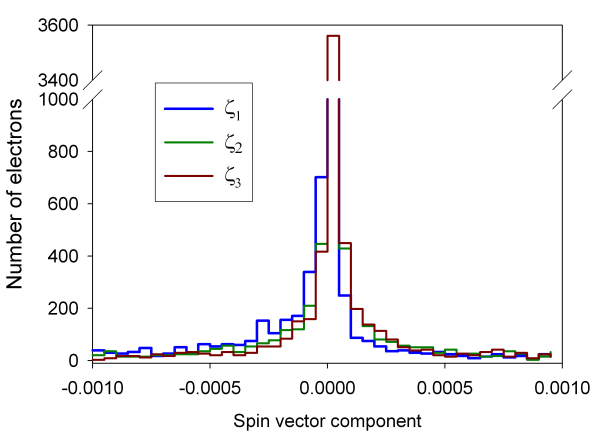

Figure 2: Polarization of final fermions. 


\section{NUMERICAL RESULTS}

Modifications of the CAIN program were kept to a miniumum by writing new analytic expressions in forms similar to those already existing in CAIN. The monte carlo scheme that determines whether a particular pair production process will take place relies on the BreitWheeler cross-section structure with respect to final electron energy and momentum $(\epsilon, p)$ (equation 4). Other required CAIN modifications were the extension of existing polarization vectors to all components, and use was made of the existing basis vector structure in CAIN.

Initial photon states, for both real and virtual particles, reveal almost no circular polarization component $\xi_{2}$ (figure 1). This is a con-

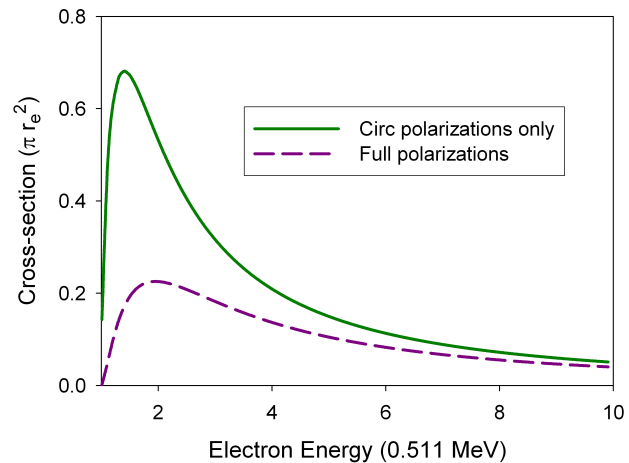

Figure 3: Breit-Wheeler cross-section. sequence of the constant, crossed beam electromagnetic field which is source of real beamstrahlung photons and with which virtual photons are identified. The components of final $e^{ \pm}$polarization vector are strongly dependent on the extent of circular polarization of initial photons. Consequently the pairs are produced with almost no polarization components (figure 2).

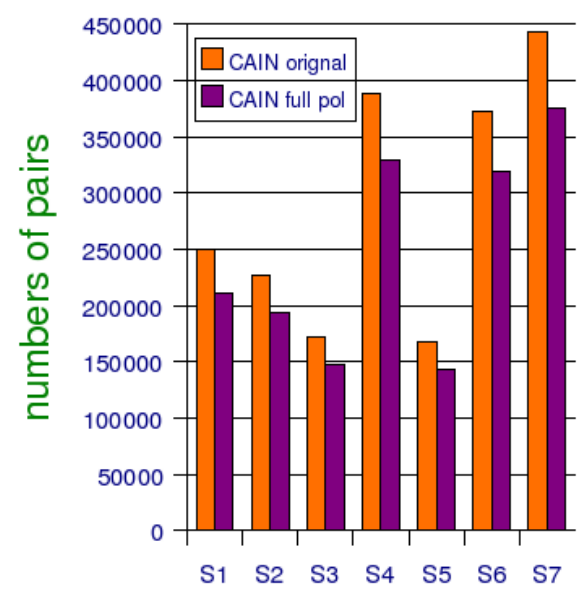

Figure 4: Pair numbers for seven parameter sets.

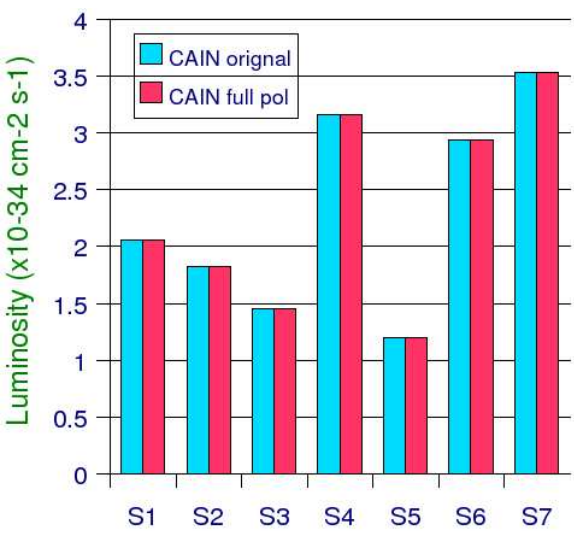

Figure 5: Luminosity for seven parameter sets.

A numerical investigation of the two cross-sections in equation 4 reveal the usual peak at low energies. However accounting for full polarizations reveals a substantially reduced cross-section for electron energies approximately less than $50 \mathrm{MeV}$ (figure 3). It was expected that such a reduction in the Breit-Wheeler cross-section would result in less background pairs. It was also considered important to determine any effect on collision luminosity. So the modified CAIN program was run for all seven $500 \mathrm{GeV}$ centre of mass collider parameter sets [4]. There was a $10-20 \%$ overall reduction in pairs (figure 4) with no discernible effect on collision luminosity (figure 5) 


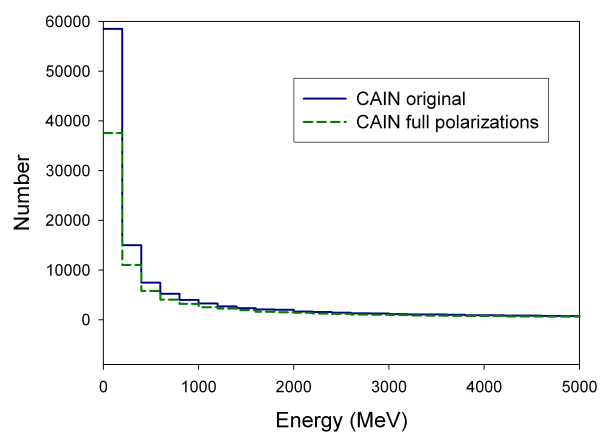

Figure 6: Energy of final pairs.

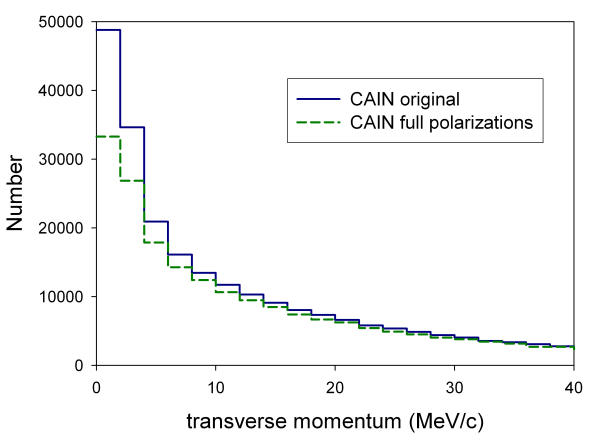

Figure 7: Transverse momentum of final pairs.

The energy and momentum of the new ensemble of expected pairs was also obtained. The shortfall in pair backgrounds was almost entirely made up of low energy and low transverse momentum pairs (figures 6 and 7).

\section{CONCLUSION}

The full polarizations of initial states, final states and the Breit-Wheeler cross-section have been investigated analytically and numerically. This enables the effect of full polarizations on three incoherent background pair processes to be determined. Real beamstrahlung photons have almost no circular polarization component. Virtual photon polarization is related to the constant, crossed bunch electric field at the point of production and likewise has no circular polarization component. The full Breit-Wheeler cross-section with all polarization states was written in similar form to the Breit-Wheeler cross-section with circular polarizations only. The CAIN program was modified and run for the seven parameter sets contemplated for the ILC with $500 \mathrm{GeV}$ centre of mass collision energy. In all cases analysis of the full polarization effects revealed that there were $10-20 \%$ less low energy background pairs than was previously thought. At the same time there was no discernible change in collision luminosity.

\section{References}

[1] CAIN Users Manual, Version 2.35 (2003)

[2] R. Engel, A.Schiller, V.G. serbo, Z Phys C, 71 (1996) 651.

[3] V.N. Baier, A.G.Grozin, hep-ph/0209361

[4] T. Raubenheimer, "Suggested ILC Beam Parameter Range" 